International Journal of Modern Physics A

(C) World Scientific Publishing Company

\title{
Heavy Antiquark Pentaquarks in the CM and GB Models
}

\author{
Kim Maltman \\ Dept. Math. and Stats, York Univ., Toronto, ON CANADA \\ CSSM, Univ. of Adelaide, Adelaide, SA, Australia
}

\begin{abstract}
The splittings between positive parity pentaquarks containing a single heavy $(c, b)$ antiquark and four light $(u, d)$ quarks are investigated in models having spin-dependent interactions generated by either effective Goldstone boson exchange or effective color magnetic exchange. Model-independent features of these splittings are emphasized. Overlaps to the "fall-apart" decay configurations $N D, N D^{*}$ or $N B, N B^{*}$, relevant to decay couplings for the various 2 -body pentaquark decays, are also computed.
\end{abstract}

\section{Introduction}

Recent experimental observations of the $S=+1 \theta$ baryon 1 , together with reports of signals for an $I=3 / 2 \Xi$ state $^{2}$ and a $C=-1$ baryon state ${ }^{3}$ have created intense theoretical interest in pentaquark states. Both chiral soliton 4 and quark mode 5678910 scenarios for the nature of these states have been investigated, but to date no clear theoretical consensus has emerged.

A key goal for model-dependent approaches is to find predictions unaffected by uncertainties in either model parameters or incompletely understood dynamical model features. For quark model approaches based on effective color magnetic (CM) or Goldstone boson (GB) exchange, we show that the pattern of splittings in the positive parity $(P=+) \bar{Q} \ell^{4}(Q=c, b, \ell=u, d)$ sectors provides several such predictions, allowing the models to be tested (and potentially ruled out) by future experiments. We concentrate on the $P=+$ sector, corresponding to relative p-wave meson-baryon decays, since, due to the centrifugal barrier, attractive short-range interactions insufficiently strong to bind can still produce resonant behavior. We study all Pauli allowed states of the form $\bar{Q} \ell^{4}$ having light quark $[31]_{L}$ orbital and $[211]_{c}$ color symmetry. (The optimal hyperfine attraction is much greater in the $[31]_{L}$ than in the $[4]_{L}$ orbital sector ${ }^{518}$.) For the explicit forms of the spatial wavefunctions, see Ref. 10. For the GB case, the spin-dependent interactions, $H_{G B}$, depend on spin and flavor (see Ref. 5 for details); for the CM case they depend on color and spin (see Ref. $\frac{10}{10}$ for details of $H_{C M}$ ).

For each $\left(I, J_{q}\right)$ (with $J_{q}$ the total intrinisic spin), $H_{G B, C M}$ are diagonalized in the basis of all Pauli-allowed states. The collection of these results yields the ground state quantum numbers and excitation spectrum. The pattern of splittings is determined solely by the structure of $H_{G B, C M}$; the overall size scales with that 
of the spatial matrix element of a relative s-wave $\ell \ell$ pair (see Ref. 10 for details).

\section{Relation to the Jaffe-Wilczek and Karliner-Lipkin Scenarios}

In the Jaffe-Wilczek (JW) scenario $\underline{6}$, the $\theta$ is assumed to consist of two $I=J_{q}=0$, $C=\overline{3} q q$ pairs coupled to $C=3$, with only confinement acting between the $\bar{s}$ and $q q$ pairs. Cross-cluster antisymmetrization (AS'n) and interaction effects are assumed suppressed by the relative p-wave between the diquark pairs. The same $\ell^{4}$ configuration is expected for the heavy pentaquark ground states, $\theta_{c, b}$. The analogous baryon splittings, $m_{\Lambda_{c, b}}-m_{\Lambda}$, are used to estimate $m_{\theta_{c, b}}-m_{\theta}$, yielding

$$
\left[m_{\theta_{c}}\right]_{J W} \simeq 2710 \mathrm{MeV}, \quad\left[m_{\theta_{b}}\right]_{J W} \simeq 6050 \mathrm{MeV},
$$

$\sim 100,170 \mathrm{MeV}$, respectively, below $N \bar{D}, N B$ thresholds.

In the GB model, the JW correlation is by far the most attractive $q q$ correlation. The model, with GB exchange acting only in the $\ell^{4}$ sector, thus corresponds to the JW scenario and provides a dynamical framework for studying (1) corrections associated with inclusion of cross-cluster AS'n/interaction effects and/or configuration mixing, and (2) the pattern of excitations above a JW-like ground state, not addressed in the JW scenario. The model yields JW ground state quantum numbers, a $\theta_{c, b} \ell^{4}$ configuration identical to that in the $\theta$, but a ground state hyperfine expectation $\sim 35 \%$ more attractive than the strict $\mathrm{JW}$ scenario value.

In the (CM-motivated) Karliner-Lipkin (KL) scenario, the $\theta$ consists of one $I=J_{q}=0, C=\overline{3}$ diquark and one $\left(I, J_{q}, C\right)=\left(0, \frac{1}{2}, 3\right)$ "triquark" (in which the spin and color of a $J_{q}=1, C=6 u d$ pair are anti-aligned to those of the $\left.\bar{s}\right)$ [ The KL correlation has lower CM hyperfine energy than does the JW correlation. The $q \bar{Q}$ interactions (responsible for favoring the triquark configuration) are weakened when $\bar{s} \rightarrow \bar{c}, \bar{b}$, reducing the hyperfine attraction for the $\theta_{c, b}$. KL estimate this reduction by assuming that (i) the $\theta_{c, b}$ and $\theta$ share the same diquark-triquark structure, and (ii) the $\bar{Q}$ hyperfine interactions scale, as in the CM model, with $1 / m_{\bar{Q}}{ }^{7}$. The resulting modified JW-style estimates are $\sim 180 \mathrm{MeV}$ above strong decay thresholds:

$$
\left[m_{\theta_{c}}\right]_{J W} \simeq 2985 \mathrm{MeV}, \quad\left[m_{\theta_{b}}\right]_{J W} \simeq 6400 \mathrm{MeV} .
$$

In the $\theta$ sector of the CM model, however, the same $\ell \bar{s}$ interactions which lower the KL triquark energy also mix the JW and KL configurations 8 (an effect also weakened when $\bar{s} \rightarrow \bar{c}, \bar{b}$ ). The $I=0, J_{q}=1 / 2$ ground state hyperfine expectation is significantly lower than either the JW or KL expectations, and well approximated using the optimized combination of JW and KL correlations 8 . For conventional values of $m_{\bar{b}, \bar{c}}$, the KL correlation is less attractive that the JW correlation in both the $\theta_{c, b}$ systems; hence the JW, and not the KL, correlation is expected to dominate these systems. The CM model ground state hyperfine expectation, including crosscluster AS'n/interaction effects neglected by KL, is, indeed, within $\sim 13 \%$ (though less attractive) of the JW correlation estimate. The resulting modified JW-style $\theta_{c, b}$ 
mass estimates lie just above strong decay threshold for the $\theta_{c}$, and just below for the $\theta_{b}$ :

$$
m_{\theta_{c}} \simeq 2835 \pm 30 \mathrm{MeV}, \quad m_{\theta_{b}} \simeq 6180 \pm 30 \mathrm{MeV} .
$$

\section{Results and Conclusions}

Results for the splittings and overlaps are given in Tables 1, 2. Column 1 lists $\left(I, J_{q}\right)$, column $2 \Delta \hat{E}$, the hyperfine splitting relative to the $\left(I, J_{q}\right)=(0,1 / 2)$ ground state, in dimensionless units 10 . The scale of the splittings in physical units is obtained by restoring an overall factor involving the hyperfine expectation for a relative s-wave $\ell \ell$ pair 10. Estimating this factor using the corresponding $N$ expectation (equivalently, using the $\Delta-N$ splitting) yields the estimated physical splitting values, $\Delta E^{e s t}$, given in Column 3. Columns 4 and 5 contain, for each excited pentaquark state $P^{*}$, the squares of the ratios $g_{P}$ and $g_{V^{*}}$, defined by

$$
g_{P}=\left\langle N P_{H} \mid P^{*}\right\rangle /\left\langle N P_{H} \mid P_{g n d}\right\rangle, \quad g_{V^{*}}=\left\langle N V_{H}^{*} \mid P^{*}\right\rangle /\left\langle N P_{H} \mid P_{g n d}\right\rangle,
$$

with $P_{\text {gnd }}$ the corresponding $\left(I, J_{q}\right)=(0,1 / 2)$ ground state, and $P_{H}, V_{H}^{*}$ the corresponding heavy pseudoscalar and vector mesons. The relative coupling strengths for the decays $P^{*} \rightarrow N M$, with $M=P_{H}, V_{H}^{*}$, should be given by the ratios of these factors if the dominant mechanism for $P=+$ pentaquark decay to $N M$ is "fall-apart" through the p-wave barrier 11.

Table 1. Low-lying positive parity excitations of the $\theta_{c, b}$ in the GB model.

\begin{tabular}{ccccc}
\hline$\left(I, J_{q}\right)$ & $\Delta \hat{E}$ & $\Delta E^{e s t}(\mathrm{MeV})$ & $g_{P}^{2}$ & $g_{V^{*}}^{2}$ \\
\hline$(0,1 / 2)$ & 0 & 0 & 1 & 3.00 \\
$(1,1 / 2)$ & $4.50 \rightarrow 5.71$ & $132 \rightarrow 167$ & $2.24 \rightarrow 2.54$ & $0.75 \rightarrow 0.85$ \\
$(1,3 / 2)$ & $4.50 \rightarrow 5.71$ & $132 \rightarrow 167$ & 0 & $1.27 \rightarrow 1.36$ \\
$(0,1 / 2)$ & $10.2 \rightarrow 14.5$ & $299 \rightarrow 423$ & $2.01 \rightarrow 2.07$ & $0.67 \rightarrow 0.69$ \\
$(0,3 / 2)$ & $10.2 \rightarrow 14.5$ & $299 \rightarrow 423$ & 0 & $2.68 \rightarrow 2.75$ \\
\hline
\end{tabular}

The tables (and extended versions thereof ${ }^{10}$ ) show the following: (1) the $P=+$ ground state has $I=J_{q}=0$ for both the CM and GB models; (2) the lowest excitation above this state is considerably lower in the CM than in the GB model; (3) the spectrum of excitations is much denser in the CM than in the GB model, $5 C=$ -1 baryons being predicted in the interval between $\sim 90$ and $130 \mathrm{MeV}$ (respectively between $\sim 150$ and $330 \mathrm{MeV}$ ) above the lowest state in the CM (respectively GB) case; (4) for the "low-lying" excitations just noted, there are no large deviations from 1 in the relative two-body decay couplings; (5) five additional excitations having rather small couplings to the two-body decay channels are also expected within $\sim m_{\Delta}-m_{N}$ of the ground state in the CM model. 
Table 2. Low-lying positive parity excitations of the $\theta_{c, b}$ in the CM model.

\begin{tabular}{lccccc}
\hline Sector & $\left(I, J_{q}\right)$ & $\Delta \hat{E}$ & $\Delta E^{e s t}(\mathrm{MeV})$ & $g_{P}^{2}$ & $g_{V^{*}}^{2}$ \\
\hline \hline Charm & $(0,1 / 2)$ & 0 & 0 & 1 & $0.74 \rightarrow 2.22$ \\
& $(0,1 / 2)$ & $1.14 \rightarrow 1.20$ & $84 \rightarrow 88$ & $0.55 \rightarrow 1.87$ & $1.54 \rightarrow 2.32$ \\
& $(1,1 / 2)$ & $1.22 \rightarrow 1.47$ & $89 \rightarrow 108$ & $1.95 \rightarrow 3.41$ & $0.03 \rightarrow 0.35$ \\
& $(0,3 / 2)$ & $1.29 \rightarrow 1.56$ & $94 \rightarrow 114$ & 0 & $1.60 \rightarrow 2.79$ \\
& $(1,3 / 2)$ & $1.61 \rightarrow 1.87$ & $118 \rightarrow 137$ & 0 & $0.85 \rightarrow 1.52$ \\
& $(1,1 / 2)$ & $1.79 \rightarrow 2.07$ & $131 \rightarrow 152$ & $0.00 \rightarrow 0.14$ & $1.72 \rightarrow 2.72$ \\
\hline \hline Bottom & $(0,1 / 2)$ & 0 & 0 & 1.00 & $1.87 \rightarrow 2.71$ \\
& $(0,1 / 2)$ & $1.16 \rightarrow 1.25$ & $85 \rightarrow 92$ & $1.54 \rightarrow 2.32$ & $0.88 \rightarrow 0.94$ \\
& $(0,3 / 2)$ & $1.26 \rightarrow 1.35$ & $92 \rightarrow 99$ & 0 & $2.51 \rightarrow 3.21$ \\
& $(1,1 / 2)$ & $1.43 \rightarrow 1.55$ & $105 \rightarrow 114$ & $1.76 \rightarrow 3.65$ & $0.20 \rightarrow 0.76$ \\
& $(1,3 / 2)$ & $1.58 \rightarrow 1.66$ & $116 \rightarrow 122$ & 0 & $1.36 \rightarrow 1.76$ \\
& $(1,1 / 2)$ & $1.77 \rightarrow 1.99$ & $130 \rightarrow 146$ & $0.05 \rightarrow 0.46$ & $2.53 \rightarrow 2.76$ \\
\hline
\end{tabular}

\section{Acknowledgements}

The support of the Natural Sciences and Engineering Research Council of Canada is gratefully acknowledged.

\section{References}

1. T. Nakano et al., Phys. Rev. Lett. 91, 012002 (2003); V. Barmin et al., Phys. At. Nucl. 66, 1715 (2003); S. Stepanyan et al., Phys. Rev. Lett. 91, 252001 (2003); J. Barth et al., Phys. Lett. B572, 127 (2003); A. E. Asratyan, A. G. Dolgolenko and M.A. Kubantsev, hep-ex/0309042 V. Kubarovsky et al., Phys. Rev. Lett. 92, 032001 (2004); A. Airapetian et al., hep-ex/0312044 A. Aleev et al., hep-ex/0401024 M. Abdel-Bary et al., hep-ex/0403011 S. Chekanov et al., hep-ex/0403051

2. C. Alt et al. (The NA49 Collaboration), Phys. Rev. Lett. 92: 042003 (2004).

3. A. Aktas et al. (The H1 Collaboration), hep-ex/0403017

4. See, e.g., D. Diakonov, V. Petrov, M. Polyakov, Z. Phys. A359, 305 (1997); H. Weigel, Eur. Phys. J. A2 (1998) 391, Eur. Phys. J. A21, 133 (2004); H .Walliser and V.B. Kopeliovich, J. Exp. Theor. Phys. 97, 433 (2003); N. Itzhaki et al., Nucl. Phys. B684, 264 (2004); V.B. Kopeliovich, hep-ph/0310071 D. Borisyuk, M. Faber, A. Kobushkin, hep-ph/0312213 D. Diakonov and V. Petrov, Phys. Rev. D69, 094011 (2004); J.R. Ellis, M. Karliner, M. Praszalowicz, JHEP 0405, 002 (2004); B. Wu and B.Q. Ma, hep-ph/0402244 M.A. Nowak, M. Praszalowicz, M. Sadzikowski and J. Wasliuk, hep-ph/0403184 and other references therein.

5. F. Stancu, Phys. Rev. D58, 111501 (1998); F. Stancu and D.O. Riska, Phys. Lett. B575, 242 (2003); F. Stancu, Phys. Lett. B595, 269 (2004) [Erratum: ibid. B598, 295 (2004)].

6. R.L. Jaffe and F. Wilczek, Phys. Rev. Lett. 91, 232003 (2003).

7. M. Karliner and H. Lipkin, Phys. Lett. B575, 249 (2003) and hep-ph/0307343

8. B. Jennings and K. Maltman, Phys. Rev. D69, 094020 (2004).

9. See also, e.g., M. Oka, Prog. Theor. Phys. 112, 1 (2004); K. Maltman, hep-ph/0408144 S.L. Zhu, hep-ph/0410002 and additional references cited therein.

10. K. Maltman, hep-ph/0408145

11. F.E. Close and Q. Zhao, Phys. Lett. B590, 176 (2004). 